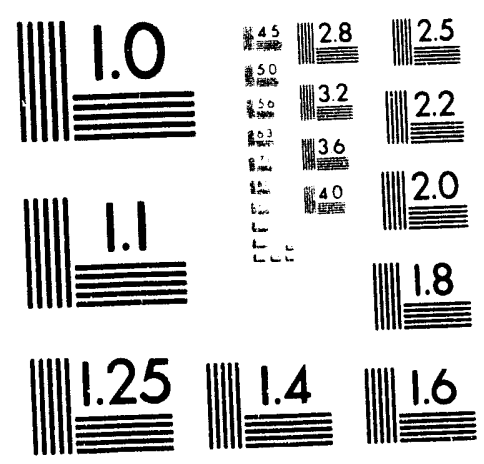



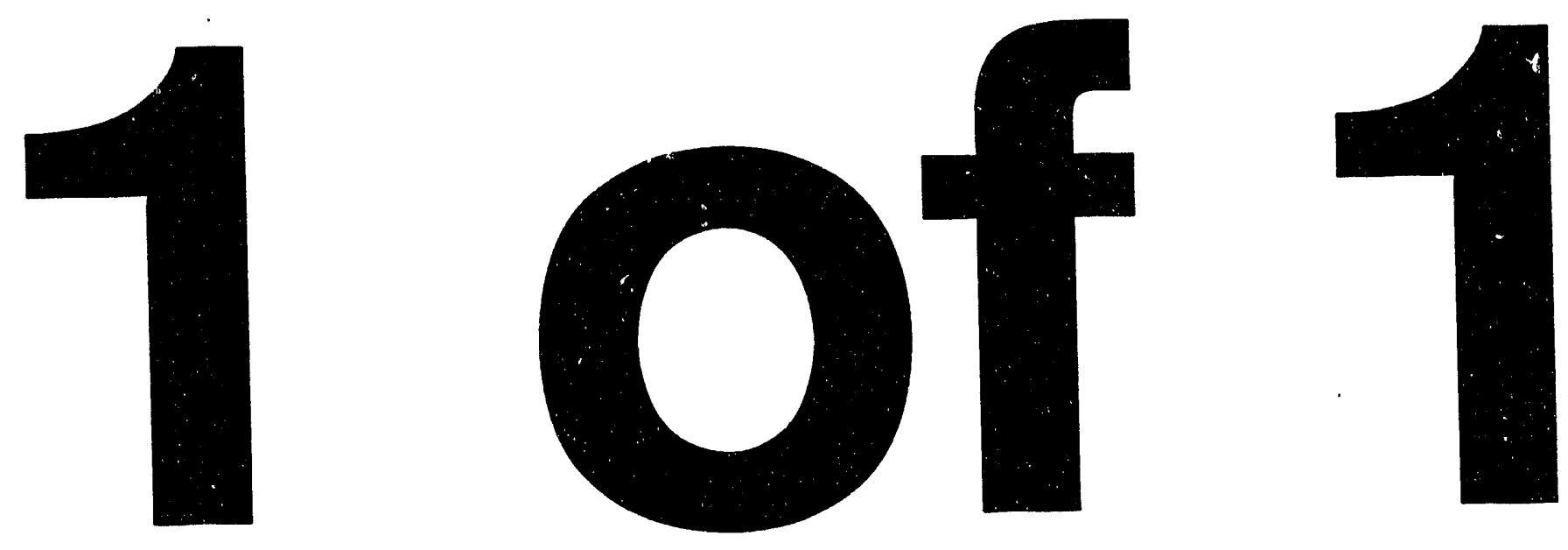
UCRL-CR- 113135

B192560

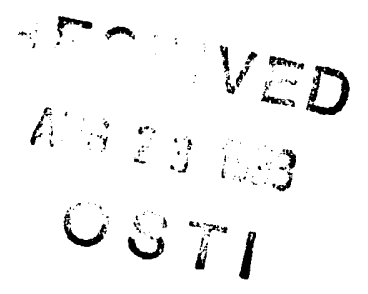

Generalized Energy Principle for Flute Perturbations in Axisymmetric Mirror Machines

I. M. Lansky and D. D. Ryutov Budker Institute of Nuclear Physics

630090, Novosibirsk, Russia

January 20, 1993

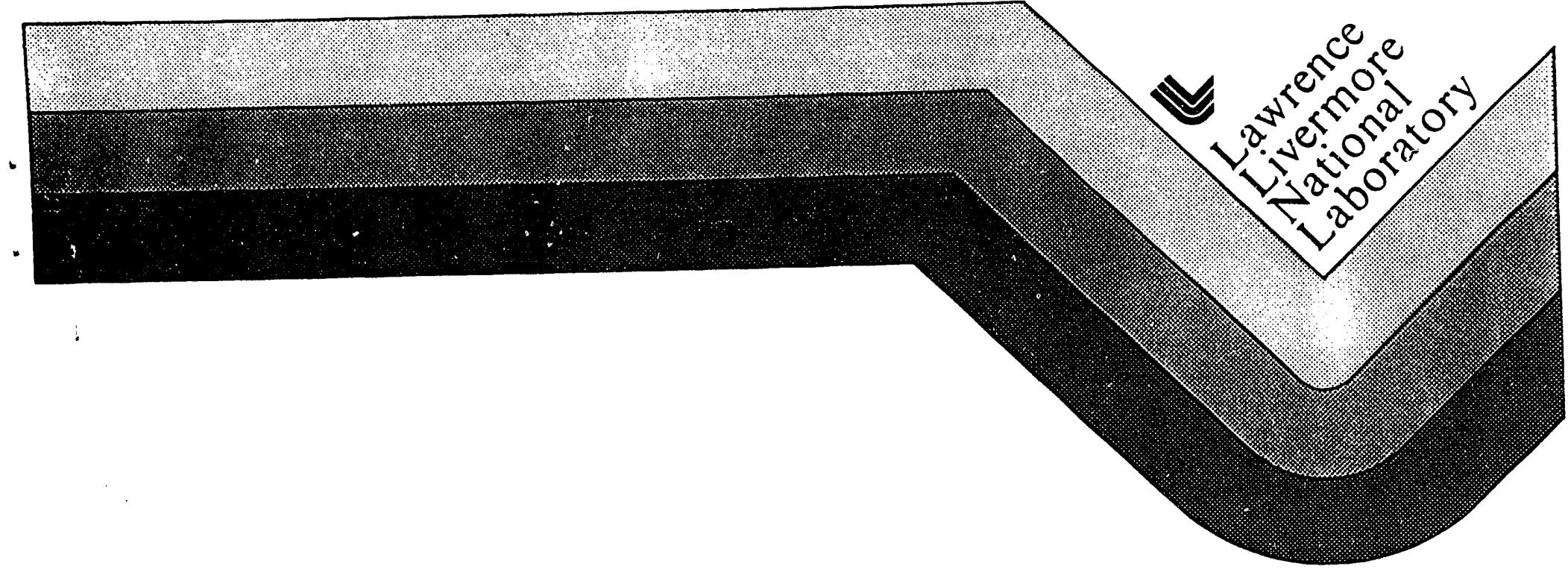

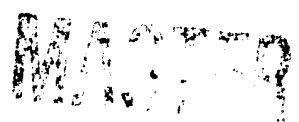

DISTRABUTION OF THIS OOCUMERT IS UNLIMITED 


\section{DISCLAIMER}

Work performed under the auspices of the U.S. Department of Energy by Lawrence Livermore National Laboratory under contract number W-7405-ENG-48.

This document was prepared as an account of work sponsored by an agency of the United Stutes Governmeat. Neither the United States Government nor the University of California nor any of their employees, makes any warranty, express or implied, or assumes any legal liability or responsibility for the accuracy, completesess, or msefulness of any information, apparatus, product, or process disclosed, or represents that its use would not iafringe privately owned rights. Reference herein to any specific commercial products, process, or service by trade name, trademark, manufacturer, or otherwise, does not necessarily constitute or imply its endorsement, recommendation, or favoring by the United States Government or the University of California. The views and opinions of authors expressed herein do not necessarily state or reflect those of the United States Government or the University of California, and shall not be used for advertising or product endorsement purposes. 


\title{
GENERALIZED ENERGY PRINCIPLE FOR FLUTE PERTURBATIONS IN AXISYMMETRIC MIRROR MACHINES
}

\author{
I.M.Lansky, D.D.Ryutov \\ Budker Institute of Nuclear Physics, \\ 630090, Novosibirsk, Russia
}

January 20, 1993

\section{Introduction}

Axial symmetry is a very desirable property of the mirror devices both for fusion and neutron source applications. The main obstacle to be circumvented in the development of such systems, is the flute instability of axisymmetric mirrors. In recent years there appeared a number of proposals, devoted to the stabilization of the flute perturbations in the framework of axisymmetric magnetic configurations, which are based on the combining of the MHD unstable central cell $\boldsymbol{F}$ ith various types of end-cell stabilizers [1]-[3]. In the present paper we concentrate ourselves just on this scheme, including long solenoid with a uniform fisld, conjugated with the end stabilizing anchor, intended to provide MHD stability of the system as a whole. The attractive feature of such a configuration is that it allows to exploit finite larmor radius (FLR) effects [4] for the stabilization of the flute perturbations. As is well known [4], FLR effects, being strong, stabilize all flute modes, except the one with azimuthal number $m=1$, corresponding to the "rigid" displacement of the plasma column (the "global" mode). Consequently, in the conditions when FLR effects dominate, the anchor has to stabilize the "global" mode only. Bearing in mind a favorable influence of FLR effects we, however, don't restrict our paper by discussion of only "global" mode stability and consider a general case of an arbitrary azimuthal mode.

The conventional approach to the stability analysis (used, for instance, in $[1,2]$ ) contains an assumption that the curvature-induced particle drift is slow as compared with the scaletime of the flute perturbation growth,

$$
\Omega_{d} \ll \Gamma,
$$


where $\Gamma$ denotes a characteristic growth-rate of the flute perturbation (or a characteristic frequency in the stable case), and $\Omega_{d}$ is a characteristic drift frequency in the stabilizing anchor. Inequality (1) means that plasma particles in the anchor undergo displacement remaining within the flute flux tube that they were initially occupying. Therefore their perturbed position can be defined by $2-D$ hydrodynamic displacement vector $\xi$, being the same for all particles, occupying in the initial state some flux tube, irrespective to their velocity and pitch angle. So, one can analyze the stability of the system in the framework of the familiar Kruskal-Oberman energy principle [5]. Just this approach was used in $[1,2]$.

On the other hand, the presence of a long central cell, possessing large inertia, can result in the violation of (1). Indeed, flute growth-rate in the central cell itself (without the anchor) scales as [6]

$$
\Gamma \sim v_{T i} / \sqrt{L_{c} L_{t r}}
$$

where $L_{c}, L_{t r}$ are lengths of solenoidal and transition regions respectively, and $v_{T i}$ is the ion thermal velocity in the central cell. It looks hardly probable to achieve a stabilizing contribution of the anchor more than 1.5 - 2 times exceeding the unstable contribution of the central cell. Hence, if one switches on the anchor, the characteristic frequency of the flute oscillations keeps its former value (2), just the sign of $\Gamma^{2}$ changes. Drift frequency around the magnetic axis in the anchor region is estimated as follows:

$$
\Omega_{d} \sim \frac{c T_{a}}{e B_{d} L_{a}^{2}}
$$

$B$, being the magnetic field in the stabilizer middle plane, $T$, being plasma temperature in the stabilizer (to avoid unnecessary notation overloading in estimates, we suppose electron and ion temperatures in the anchor to be equal to each other). As it follows from (2),(3), growth-rate $\Gamma$ becomes really small as compared with the drift frequency when $L_{c}$ is large enough.

The latter conclusion gives rise to the interest for the investigation of the situation when the drift frequency in the stabilizing anchor exceeds essentially the frequency of MHD perturbations, i.e. when inequality inverse to (1) is satisfied:

$$
\Omega_{d} \gg \Gamma \text {. }
$$

To analyze the stability of such a system one cannot use Kruskal-Oberman energy principle and has to rely on its modified version that takes into account the condition (4).

The generalized energy principle accounting for the contribution of nonhydrodynamic (in the sense of (4)) plasma species has been first formulated in [7], where single-particle adiabatic invariant technique has been used to calculate the perturbation of the particle energy. The energy variation, derived in [7], consists of the contributions of both perturbed plasma and magnetic energies. However, if one inserts into the expression for $W$, presented in [7], the displacement vector $\boldsymbol{\xi}$ of the flute perturbation

$$
\boldsymbol{\xi}=\frac{\nabla \eta \times \mathbf{B}}{B^{2}}
$$


with $\eta$ constant along the field line, then one comes to a somewhat paradoxical result. Namely, it turns out that $W$, as given in [7], becomes identically zero (see Appendix $A$ ). The reason is that authors of paper [7] retained in $W$ only terms proportional to $\xi^{2}$ while, as it has been shown in [8], the contribution of non-MHD particles to the energy of the perturbations has a different structure, being proportional to $\dot{\xi}^{2}$. Henceforth in this paper we consider just the case of purely flute perturbations (5).

Now it's appropriate to mark one more aspect. The stabilization due to strong FLR effects, mentioned above, takes place in the conditions [4]

$$
\Gamma \ll \frac{c T_{c}}{e B_{c} a_{c}^{2}}
$$

where $B_{c}, T_{c}, a_{c}$ are magnetic field, temperature and plasma radius in the central cell. Combining (1), (3), (6) and taking into account the conservation of the magnetic flux through the plasma cross-section,

$$
B_{\mathrm{c}} a_{c}^{2} \sim B_{s} L_{s}^{2}
$$

(here we suppose that radial dimension of plasma in the stabilizer is of the same order of its axial dimension $L_{0}$ ) one can find that the assumption of the dominating FLR effects in the limit (1) is valid only if

$$
T_{\text {. }} \ll T_{c} \text {. }
$$

Since the latter condition is rather restrictive, it stresses all the more the actuality of the stability analysis, involving particles with fast drift frequency (4).

The contents of the present paper is as follows. In the next Section we reproduce the derivation of the generalized energy principle [8] for the axisymmetric case. In Section 3 we invoke the cited energy principle to illustrate the possibility of the stabilization of the flute instability due to the "negative inertia" effect. The investigation of the conditions, required for the "negative inertia" stabilization in the magnetic mirror, is the subject of Section 4. Section 5 is devoted to the application of the generalized energy principle to stability analysis of the neutron source devicc, proposed in [9]. Section 6 contains conclusions. Several computational subjects are carried out in Appendices. In particular, in Appendix $\mathrm{C}$ we show that the energy principle [8] admits the generalization to the intermediate frequency interval, $\Gamma / m \ll \Omega_{d} \leq \Gamma$, for modes with high azimuthal numbers $m$.

\section{Generalized energy principle for axisymmetric plasma configurations}

Suppose that in the anchor region there exists a population of hot particles with drift frequencies satisfying inequality (4), while other plasma both in the anchor and in the central cell is cold enough to admit conventional MHD treatment. We examine the stability of such a system with respect to the purely electrostatic perturbations that are characterized by the electrostatic potential $\varphi$, constant along the field line. The latter assumption, identical to 
the one made in [7], implies the presence of a cold plasma component which justifies the constancy of the potential along the field line and acts to provide quasineutrality condition.

In this paper we adopt the following coordinate system: we mark every field line with the polar coordinates $r, \psi$ of its intersection with some plane, perpendicular to the uniform magnetic field in the central solenoid. Instead of $r$ one can use the magnetic flux $\Phi$ inside the cylindrical surface of the radius $r: \Phi=\pi r^{2} B_{c}$. A drift surface can then be described by the equation $\Phi=\Phi(\psi)$. In the introduced coordinates potential $\varphi$ of the perturbations, corresponding to the mode with azimuthal number $m$, is given by

$$
\varphi=\tilde{\varphi}(\Phi) \cos m \psi
$$

Note that

$$
\bar{\varphi}(0)=0,
$$

since there is no azimuthal component of the electric field or the magnetic axis.

At a given configuration of magnetic and electrostatic fields, the drift surface for a particle with a total energy $\varepsilon$ and magnetic moment $\mu$ is determined by the constancy of the longitudinal action

$$
J(\varepsilon, \mu, \Phi, \psi)=(2 M)^{1 / 2} \int(\varepsilon-\mu B-e \varphi)^{1 / 2} d l
$$

with the integration carried out between the turning points. If the condition (4) is satisfied, then, with the electrostatic potential varying, the drift surface adjusts itself to keep constant the magnetic flux inside the surface [10]. This occurs via variation of the particle energy.

To find the change $W$ of the kinetic energy of the particles (just this quantity enters the energy principle for the perturbations with a scale-length much in excess of the Debye radius), we use the following approach. We consider some group of particles (of a total number $\Delta N$ ) that in the initial state have certain values of $\varepsilon$ and $\mu$, and that are filling a drift surface characterized with a certain value of $J$. When we slowly turn on the electrostatic potential of the perturbation, the drift surface deforms and the kinetic energy of the particles changes. If we find the change of the kinetic energy $د W^{\prime}$ for this group, then, by summation over all the groups, we find the required quantity $I F$.

The group $\Delta N$ is drifting along the contour $\Phi(\dot{v})$ determined by the instantaneous configuration of the electrostatic field and the instantaneous value of $\varepsilon$. The number of particles from this group $d \Delta N$, occupying the section of the contour of the arc length $d \psi$, can be presented in the form $d \Delta N=\nu d \psi$, where $\nu$ is the number of particles per unit arc length. The stationarity condition $\nu \dot{\psi}=$ const yields:

$$
\nu=\frac{\Delta N \Omega_{d}}{2 \pi i},
$$

where $\dot{\psi}$ is the angular velocity of the bounce-averaged drift motion $[11]$ :

$$
\dot{\psi}=-\frac{2 \pi c}{e t_{i}} J_{\phi},
$$


$t_{\|}=J_{c}$ is the transit time between the turning points, and $\Omega_{d}$ is the drift frequency,

$$
\Omega_{d}=2 \pi\left[\int_{0}^{2 \pi} \frac{d \psi}{\dot{\psi}}\right]^{-1}
$$

We use notations $J_{\epsilon} \equiv \partial J / \partial \varepsilon, J_{\Phi} \equiv \partial J / \partial \Phi$, etc., for the partial derivatives. The change of the kinetic energy of these particles is, obviously,

$$
\Delta W=\left.\left[\int_{0}^{2 \pi} \nu(\varepsilon-e \varphi) d \psi\right]\right|_{\text {initial }} ^{\text {final }},
$$

where the subscripts indicate the difference between the final and initial state. The total energy $\varepsilon$ of particles is constant over the drift surface in the time-scale of $\Omega_{d}{ }^{-1}$, while their kinetic energy $\varepsilon-e \varphi$ varies from one field line to another according to variation of $\varphi$. In this respect, $\Delta W$, if divided by $\Delta N$, represents the average (over the drift eurface) change of the kinetic energy.

The condition of flux conservation inside the drift surface can be written in the form:

$$
\left.\int_{0}^{2 \pi} \Phi(\psi) d \psi\right|_{\text {initial }} ^{\text {final }}=0
$$

where $\Phi(\psi)$ is a solution of the equation

$$
\left.J(\varepsilon, \mu, \Phi, \psi)\right|_{\text {initial }} ^{\text {final }}=0 .
$$

In principle, equations (11)-(16) allow one to find the particle kinetic energy at arbitrarily large $\varphi$. However, we will consider only the case of small $\varphi$. The quantities of the first order in $\varphi$ will be denoted with subscript " 1 ", the second order corrections by subscript " 2 ", etc.

In the linear approximation, equation (16) yields:

$$
\left(\varepsilon_{1}-e \varphi\right) J_{e}+\Phi_{1} J_{\Phi}=0
$$

wherefrom, taking into account relationships (8) and (15), we find that

$$
\varepsilon_{1}=0, \quad \Phi_{1}=e \varphi \frac{J_{\varepsilon}}{J_{\Phi}}
$$

The next order expansion of (16) gives:

$$
J_{e}\left(\varepsilon_{2}-e \Phi_{1} \varphi_{\Phi}\right)+J_{\Phi} \Phi_{2}+\frac{1}{2} J_{e \varepsilon}(e \varphi)^{2}-J_{e \Phi} \Phi_{1} e \varphi+\frac{1}{2} J_{\Phi \Phi} \Phi_{1}{ }^{2}=0
$$

The requirement (15), when applied to $\Phi_{2}$, yields:

$$
\int_{0}^{2 \pi} d \psi\left(\varepsilon_{2}-e \Phi_{1} \varphi_{\Phi}\right)=-\int_{0}^{2 \pi} \frac{d \psi}{J_{\epsilon}}\left(\frac{1}{2} J_{e \varepsilon} \varphi^{2}-J_{e \Phi} \Phi_{1} e \varphi+\frac{1}{2} J_{\Phi \Phi} \Phi_{1}{ }^{2}\right)
$$


while from (14) we find that

$$
\Delta W=\frac{\Delta N}{2 \pi} \int_{0}^{2 \pi}\left[\frac{\dot{\psi}_{1}}{\dot{\psi}} e \varphi+\left(\varepsilon_{2}-e \Phi_{1} \varphi_{\Phi}\right)\right] d \psi
$$

From equation (12) one obtains that

$$
\frac{\dot{\psi}_{1}}{\dot{\psi}}=-\frac{J_{e}}{J_{\Phi}} e \varphi_{\Phi}+\frac{J_{\epsilon}}{J_{\Phi}}\left(\frac{J_{\Phi \Phi}}{J_{\Phi}}-\frac{2 J_{e \Phi}}{J_{\epsilon}}+\frac{J_{e \varepsilon} J_{\Phi}}{J_{c}^{2}}\right) e \varphi .
$$

Now, using relationships (8), (18), (21) and (22), we can express $\Delta W$ in terms of $\tilde{\varphi}$ :

$$
\Delta W=-\frac{1}{4} e^{2} \Delta N\left[\frac{J_{e}}{J_{\Phi}} \frac{\partial \tilde{\varphi}^{2}}{\partial \Phi}+\tilde{\varphi}^{2}\left(2 \frac{J_{e \Phi}}{J_{\Phi}}-\frac{J_{e \varepsilon}}{J_{e}}-\frac{J_{e} J_{\Phi \Phi}}{J_{\Phi}^{2}}\right)\right]
$$

To perform the summation over the plasma particles, we introduce the distribution function $F(\varepsilon, \mu, \Phi)$, normalized according to the relationship $\Delta N=F(\varepsilon, \mu, \Phi) \Delta \varepsilon \Delta \mu \Delta \Phi$. Then, the energy $W$ of the perturbation acquires its final form:

$$
\begin{aligned}
W=-\frac{1}{4} e^{2} \int d \varepsilon d \mu d \Phi F(\varepsilon, \mu, \Phi) \times & \\
& \times\left[\frac{J_{e}}{J_{\Phi}} \frac{\partial \tilde{\varphi}^{2}}{\partial \Phi}+\bar{\varphi}^{2}\left(2 \frac{J_{\varepsilon \Phi}}{J_{\Phi}}-\frac{J_{\epsilon \varepsilon}}{J_{\epsilon}}-\frac{J_{\varepsilon} J_{\Phi \Phi}}{J_{\Phi}^{2}}\right)\right] .
\end{aligned}
$$

Sometimes it's more convenient to use the expression for $\mathrm{II}^{\circ}$ in the form

$$
\begin{aligned}
W=\frac{1}{4} e^{2} \int d \varepsilon d \mu d \Phi \tilde{\varphi}^{2} \times & \\
& \times\left[\frac{\partial}{\partial \Phi}\left(F \frac{J_{e}}{J_{\Phi}}\right)-F\left(2 \frac{J_{e \Phi}}{J_{\Phi}}-\frac{J_{e \varepsilon}}{J_{e}}-\frac{J_{e} J_{\Phi \Phi}}{J_{\Phi}^{2}}\right)\right],
\end{aligned}
$$

which can be obtained from (24) through the integration by parts of the first term in the square brackets (24). There is no contribution of limits from the integration by parts in (25), since on the upper limit there are no particles, and so the distribution function equals to zero, while on the lower limit the potential $\varphi$ vanishes according to (9).

In Appendix $B$ we derive also the expression for the energy variation, relevant to the conditions when the longitudinal energy of the particles is small as compared with their transverse energy. In such a case plasma is located near the point of the minimum field strength on the given field line (the so-called "disk"-like plasma), and the contribution of the particles with fast drift can be expressed as

$$
W=\frac{e^{2}}{4} \int d \mu d \Phi \frac{F}{\mu} \frac{d}{d \Phi}\left(\frac{\varphi^{-2}}{B_{0 \Phi}}\right)
$$

or after the integration by parts

$$
W=-\frac{e^{2}}{4} \int d \mu d \Phi \frac{\bar{\varphi}^{2}}{\mu B_{0 \Phi}} \frac{\partial F}{\partial \Phi} .
$$

where $B_{0}$ is the minimum value of the field strength on the magnetic surface, corresponding to the flux $\Phi$, and $B_{0 \Phi} \equiv \partial B_{0} / \partial \Phi$. 


\section{Stability of the "global" mode}

Formulae (24), (25) define the contribution of only fast drifting paricles (4) in the total energy variation, while there exist two more terms, originated from plasma particles with small drift frequencies (1), whose contribution can be obtained in the framework of MHD approach. The first one scales as

$$
W^{(k)} \cong M_{i} n_{c} a^{2} L_{c} \dot{\xi}^{2}
$$

Henceforward in this section we consider the case of the "global" mode perturbations with $\xi$ as a displacement of the plasma column as the whole in the central cell. Expression (28) describes the kinetic energy of the perturbations. It is caused by inertia of ions in the long central cell.

The second term,

$$
W^{(p)} \cong-\frac{n_{c} T_{c} a^{2}}{L_{t r}} \xi^{2}
$$

describes the potential energy of the perturbations caused by the field line curvature. The sign of $W^{(p)}$ is negative due to the unfavorable field line curvature in the transition region. As it follows from (28), (29), in the central cell itself (with the anchor being switched off) the instability grows up with the characteristic increment given by (2).

Taking into consideration the contribution (24) of the fast particles, notice that the expression for $W$ scales as $\varphi^{2}$. Sir.ce the displacement $\xi$ of the flux tube, filled with a cold plasma, is determined by formuis (5) with $\eta=-(1 / c) \int \varphi d t$, we see that, if (25) is expressed in terms of $\xi$, it scales as

$$
W \cong \frac{n_{\varepsilon} L_{a}^{3}}{T_{\bullet}} \frac{e^{2} a^{2} B_{c}^{2}}{c^{2}} \dot{\xi}^{2}
$$

Here $w e$ have supposed the radial dimension of plasma in the stabilizer to be of the order of $L_{a}$, and also condition (7) has been taken into account. Thus $W$ scales as a kinetic energy of the perturbations $\left(\sim \dot{\xi}^{2}\right)$, giving contribution to $W^{(k)}$, not to $W^{(p)}$. Therefore the presence of the fast drifting particles manifests itself in changing of the "inertia" of the perturbations, not of their "rigidity".

Fast drifting particles may affect the stability in two different ways, depending on the sign of the energy $W$. Positive value of $W$ gives rise to the increasing of the effective kinetic energy of the perturbations (or of their effective "inertia") that, in turn, leads to the decreasing of the increment $\Gamma$, but the instability still remains.

It seems to be more attractive another situation, when $W$ is negative and, moreover, the following requirement is satisfied:

$$
W^{(k)}+W<0
$$

The negative sign of the effective kinetic energy corresponds to the oscillations of the perturbations with 'the 'negative inertia" in a hump-like potential (29). Since the frequency of such oscillations is, obviously, real, condition (31) allows one to conclude that the system becomes stable. 
Estimates (28), (30), together with (31), impose one more constraint on the plasma parameters:

$$
\frac{n_{s}}{n_{c}} \frac{L_{c}}{L_{c}}>\frac{\rho_{H_{s}}^{2}}{L_{s}^{2}} \frac{B_{c}^{2}}{B_{c}^{2}}
$$

where $\rho_{H}$, is the ion larmor radius of the hot particle in the stabilizer region.

To cite one more example of the magnetic configuration in which the fast drifting particles may considerably affect the stability of the plasma we consider a single non-paraxial mirror of length $L$ (with a plasma occupying a volume of the order of $L^{3}$ ). Let plasma consist of a thermal population with temperature $T$ and density $n$, and a hot population with temperature $T_{*}$ and density $n_{*}<n$; let also the pressure of the hot component exceed that of the cold one: $n_{*} T>n T$. For the mode of a "global" displacement one can evaluate the plasma kinetic energy (per unit volume) as

$$
\left(\frac{n \cdot T_{*}}{\Omega_{d}^{2} L^{2}}+n M\right) \dot{\xi}^{2}
$$

where $\xi$ is a (small) plasma displacement. The first term here represents a contribution of the fast particles. The potential energy is just

$$
n T(\xi / L)^{2},
$$

as fast particles do not contribute to it. If the drift frequency of the fast particles $\Omega_{d}$ is not too high,

$$
\Omega_{d}<\frac{1}{\zeta}\left(\frac{n . T_{*}}{n M}\right)^{1 / 2}
$$

the inertia of the fast particles dominates. The estimate for the growth-rate $\Gamma$ is then

$$
\Gamma \sim \Omega_{d}^{\prime}\left(\frac{n T}{n_{*} T_{*}}\right)^{1 / 2}
$$

As $n_{*} T_{*}>n T$ the growth-rate is automatically less than the drift frequency, ensuring the applicability of our analysis. So, we see that, indeed, the "inertia" of the fast drifting particles can be dominant, despite their small density.

Notice that though in the present section we have concerned ourselves with the stability analysis of the "global" mode (estimates (28), (29), (32),(33) hold just for the "global" mode perturbations), the stabilization due to the "negative inertia" effect, discussed above, makes it possihle to suppress, in principle, the instability of an arbitrary azimuthal mode. However, as the "inertia" of the oscillations is negative (see (31)), the dissipative instabilities are possible.

\section{4 "Negative inertia" stabilization in the magnetic mir- ror}

As it was established in Section 3, the necessary condition for the stabilization of the flute instability due to the "negative inertia" effect is the negative definition of the energy variation 
$W$ of the fast particles:

$$
W<0 \text {. }
$$

In the given magnetic field the latter inequality imposes certain restrictions on the possible profiles of the distribution function $F$ of the fast particles. In the present Section we investigate the requirements, which the distribution function $F$ should fit to satisfy (34) in the magnetic mirror configuration.

We start with the stability analysis of the paraxial magnetic mirror. In the framework of the paraxial approach, the plasma radial dimension should be small compared to the mirror-to-mirror distance. If we use the coordinate frame with the axis coinciding with the magnetic axis, the longitudinal invariant $J$, up to the terms linear in $\Phi$, can be written as $[12]$

$$
J=J^{(0)}(\varepsilon, \mu)+\Phi J^{(1)}(\varepsilon, \mu) .
$$

The neglect of the higher order terms in $\Phi$ is justified in the paraxial region. Since the variation of $F$ in $\Phi$ has a small scale-length, the first term in (25) is dominant, and so expression for $W$ reduces to a simplified form:

$$
W=\frac{1}{2} e^{2} \int d \varepsilon d \mu d \Phi \frac{J_{\varepsilon}^{(0)}}{J^{(1)}} \tilde{\varphi}^{2} \frac{\partial F}{\partial \Phi} .
$$

Since the derivative $\partial F / \partial \Phi$ defines the sign of the diamagnetic frequency, one can conclude from (12), (35), taking into account $J_{e}^{(0)}>0$, that the energy variation would be negative for those particles whose directions of the curvature-induced and diamagnetic drifts coincide, and would be positive in the opposite case.

As an example of the stability analysis of the concrete plasma configuration, we examine the stability of the hot "disk"-like plasma located in the middle plane of the magnetic mirror. The energy variation of such a plasma is given by formulae (26), (27). The paraxial expansion of the magnetic field in the middle plane can be presented in the form [12]:

$$
B_{0}=B-\frac{1}{4 \pi} \frac{\mathcal{B}^{\prime \prime}}{B} \Phi
$$

where $B$ is the field strength $\rightarrow$ the axis and prime denotes the derivative along the axis. Note that $B^{\prime \prime}>0$, since the magnetic field has a minimum in the middle plane. Inserting (36) into (27), one obtains:

$$
W=\pi e^{2} \frac{B}{B^{\prime \prime}} \int d \mu d \Phi \frac{\bar{\varphi}^{2}}{\mu} \frac{\partial F}{\partial \Phi}
$$

As it follows from the latter expression, condition (34), necessary for the stabilization, is satisfied for a descending plasma profile,

$$
\frac{\partial F}{\partial \Phi}<0
$$


Now we investigate in more detail the stability of the "global" mode for the discussed above plasma configuration. The potential $\varphi$, corresponding the "global" mode perturbations, is given by

$$
\varphi=\varphi_{0} \sqrt{\Phi} \cos \psi
$$

After the substitution of (36), (37) into (26), one can find

$$
W=-\pi e^{2} \frac{B}{B^{\prime \prime}} \int d \mu d \Phi \frac{F}{\mu} .
$$

Inequality (34) is satisfied in this case, and so the "negative inertia" stabilization is realizable.

Up till now in the present Section the discussion has been restricted by the framework of the paraxial plasma configurzions. To illustrate the influence of non-paraxiality on the stability of the plasma with fast particles, we turn to the stability analysis of the hot "disk"like plasmá, localized in the middle plane of the magnetic mirror composed of two equal co-exis magnetic dipoles (fig.1). The magnetic field in the middle plane of such a mirror can be written in the form

$$
B_{0}=B \cdot \frac{(2-x)}{2(x+1)^{5 / 2}},
$$

where $x=4 r^{2} / L^{2}, r$ is a radius in the middle plane, $L$ is a distance between dipoles and $B$. is the field strength in the middle point between them. The magnetic flux can be expressed in terms of $x$ as follows:

$$
\Phi=\Phi, \frac{3^{3 / 2}}{2} \frac{x}{(1+x)^{3 / 2}},
$$

where $\Phi$, denotes the flux, corresponding to the separatrix that bounds the region of the adiabatic confinement (see fig.1).

Now we examine the sign definition of the energy variation $W$ for the "global" mode perturbation (37). Consider the plasma envelope, involving particles located on the distance $R_{0}$ from the axis in the equilibrium state. Inserting (37), (39), (40) into (26), after elementary analysis one can obtain, that the energy variation $W$ occurs to be negative for the plasma envelopes with radii

$$
\frac{R_{0}}{R_{2}}<0.72 \text {, }
$$

where $K_{s}$ is the separatrix radius, while for radii $R_{0}$, being outside the interval (41), the energy $W$ has a positive sign. Hence, ron-paraxial effects lead to the violation of the condition (34), and so whe region of distant radii pays unfavorable contribution to $W$ in the sense of the possibility of the "negative inertia" stabilization.

\section{Application to the beam-plasma neutron source}

Our results can be of some interest for the development of the beam-plasma neutron source (BPNS) [9]. In this system a relatively short mirror machine is filled with a cold tritium 
plasma which serves as a target for high energy ( $\sim 60 \mathrm{keV}$ ) deuterons which are produced by NB injection and confined in the same mirror machine. In order to reduce the heat losses through the cold plasma electrons, there is envisaged the use of the long solenoicisl sections with gradually decreasing magnetic field between the mirror cell and the end-walls: as the target plasma is a collisional one and the heat flux is determined by the thermal conductivity, this arrangement indeed reduces the heat flux. Another implication of the using of these long sections is a considerable increase of the inertia of the flute perturbations. As one can easily show [13], for the global mode the kinetic energy can be evaluated as

$$
\dot{\xi}^{2} \pi a_{0}^{2} B_{0}^{2} \int \frac{M n d l}{B^{2}},
$$

where $\xi$ is a plasma displacement in the equatorial plane of the device, $B_{0}$ and $a_{0}$ are magnetic field strength and plasma radius in this plane, respectively. Denoting the half-length of the mirror cell as $L$, one can obtain the following expression for the potential energy:

$$
\xi^{2}\left(n \cdot T_{*} / L^{2}\right) \pi a_{0}^{2} L
$$

where the subscript "*" refers to the high energy particles.

The growth-rate $\Gamma$ of the flute perturbations is equal to

$$
\frac{1}{L}\left(\frac{T_{*}}{M}\right)^{1 / 2}\left(\frac{n_{*} L}{B_{0}^{2} \int \frac{n d l}{B^{2}}}\right)^{1 / 2}
$$

while the drift frequency of the fast ions is

$$
\Omega_{d} \sim \frac{\rho_{*}}{L^{2}}\left(\frac{T_{*}}{M}\right)^{1 / 2}
$$

Here $\rho$. denotes the Larmor radius of the hot particle. After the substitution of the numerical values of all variables (see [9]), it turns out that

$$
\frac{\Gamma}{\Omega_{d}} \sim 0.1
$$

that is, the contribution of the fast ions to the potential energy of the flute perturbations in fact is zero. This should allow the change of the magnetic configuration from Yin-Yang to axisymmetric one, with the corresponding simplification of design of the neutron source.

\section{Conclusions}

In the present paper we have analyzed the stability of the axisymmetric mirror device with respect to purely electrostatic flute perturbations. We have investigated the scheme consisting of the long central cell conjugated with the end stabilizer that contains hot plasma. 
One of the main results of our work is the establishing of the fact, that a population of hot particles with fast azimuthal drift (see (4)) may pay a favorable contribution to the stability of the system as the whole. The reason is that fast drifting particles affect not potential energy of MHD perturbations but rather their kinetic energy, and in the case, when the effective kinetic energy is found to be negative, the stabilization takes place.

Another system, to which our results can be applied, is a two-component plasma consisting of a cold dense background and a minority of hot particles that determines the plasma pressure (like in some versions of mirror-based neutron sources [9,17]). At high enough density of a cold compozent, condition (4) can easily be met.

We have also showed that the energy principle, formulated in [8], can be generalized to the intermediate frequency interval $\Gamma / m \ll \Omega_{d} \leq \Gamma$ for the azimuthal modes with high numbers $m$ (see Appendix $C$ ). Therefore all conclusions, concerning the stabilization due to the "negative inertia" effect, can be transformed to these conditions as well.

\section{Acknowledgments}

This research was supported in part by the US Department of Energy under the Contract No B 192560 with Lawrence Livermore National Laboratory.

\section{Appendices}

\section{A Van Dam-Rosenbluth-Lee energy principle in the case of the flute perturbations}

We start with the introducing the Clebsh coordinates $(\alpha, \theta)[15]$ with property

$$
\mathbf{B}=\nabla \alpha \times \nabla \theta
$$

The $\alpha$ coordinate is chosen so that the contour surfaces of constant $\alpha$ form a nested series of topological cylinders, and it is normalized to enclose the magnetic flux $2 \pi \alpha$ by any $\alpha$ surface. The $\theta$ coordinate is angle-like and of period $2 \pi$ on each $\alpha$ surface. In the limit of zero 3 the magnetic field satisfies equation $\nabla \times \mathbf{B}=0$, and hence it can be expressed as a gradient of some potential $\chi$ :

$$
\mathbf{B}=\boldsymbol{\nabla} \chi
$$

Vectors

$$
(\nabla \alpha, \nabla \theta, \nabla \chi)
$$

compose a covariant basis that we are going to deal with. We also define a contrvariant basis $(\mathbf{u}, \mathbf{v}, \boldsymbol{\tau})$, dual to $(43)$, in such a way that

$$
\mathbf{u}=\frac{\nabla \theta \times \nabla \chi}{B^{2}}
$$




$$
\begin{gathered}
\mathbf{v}=-\frac{\nabla \alpha \times \nabla \chi}{B^{2}}, \\
\tau=\frac{\nabla \alpha \times \nabla \theta}{B^{2}} \equiv \frac{\mathbf{b}}{B} .
\end{gathered}
$$

According to the energy principle [7], derived within the assumption of the fast particle drift (see condition (4)), the energy variation $W$ consists of two terms:

$$
W=W_{f}+W_{k} \text {. }
$$

The first one, $W_{f}$, represents the local part of $W$ and can be written in the Taylor-Hastie form [14] :

$$
\begin{gathered}
W_{f}=\frac{1}{2} \int d^{3} x\left[\sigma Q_{\perp}^{2}+\zeta Q_{\|}^{2}+\sigma j_{\|} \mathbf{b} \cdot(\xi \times \mathbf{Q})+q \xi \cdot \nabla^{\prime} p_{\|}\right. \\
\left.-(1 / B)\left(2 Q_{\|}+\xi \cdot \nabla B\right)\left(\xi \cdot \nabla^{\prime} p_{\perp}\right)\right] .
\end{gathered}
$$

Here $\boldsymbol{\xi}$ is the displacement vector, $\mathbf{Q}$ is the Eulerian magnetic field perturbation,

$$
\mathbf{Q}=\boldsymbol{\nabla} \times(\boldsymbol{\xi} \times \mathbf{B})
$$

the subscripts $\|, \perp$ refer to the parallel and perpendicular components with respect to the direction of the unperturbed magnetic field, and the coefficients $\sigma$ and $\zeta$,

$$
\sigma=1-B^{-1}\left(\partial p_{\|} / \partial B\right), \quad \zeta=1-\left(\partial^{2} p . / \partial B^{2}\right),
$$

are measures of stability against firehose and mirror anisotropy modes, respectively. Also, the following notations are introduced:

$$
\begin{gathered}
\nabla^{\prime}=\nabla-(\nabla B) \partial / \partial B . \\
\boldsymbol{q}=\mathbf{b b}: \nabla \xi .
\end{gathered}
$$

The kinetic contribution to the energy variation. $11 ;$. uriginated from the fast drifting particles, is given by

$$
W_{k}=-\frac{1}{2} \int d \alpha d \theta d \mu d J\left[( \frac { \partial F } { \partial \varepsilon } ) _ { J } \left\langle\left\langle H^{2}-\left(\frac{i J}{i \xi}\right)\left(\frac{\partial F}{\partial J}\right)_{c} H_{i}^{2}\right],\right.\right.
$$

where

$$
H=-m v_{\|}^{2} q-\mu B(\nabla \cdot \xi-q) .
$$

and $F=F(J(\alpha, \theta, \varepsilon, \mu), \varepsilon, \mu)$ is the equilibrium distribution function. depending only on the integrals of motion (see e.g. [16]). Single angle brackets in $(4 i)$ describe the bounce average:

$$
\langle\ldots\rangle=\left(\frac{\partial J}{\partial \varepsilon}\right)^{-1} f d l_{r^{-i}}(\ldots) .
$$


while double angle brackets denote the average both over bounce and drift motions:

$$
\langle\langle\ldots\rangle\rangle=\left(\frac{\partial \tilde{\Phi}}{\partial \varepsilon}\right)_{J}^{-1} \oint d \theta\left(\frac{\partial \varepsilon}{\partial \alpha}\right)_{J}^{-1}\langle\ldots\rangle,
$$

where $\bar{\Phi}=\oint d \theta \alpha(\theta)$ is the flux adiabatic invariant $(\alpha=\alpha(\theta)$ defines the particle drift-surface, and integration is performing with $J$ and $\varepsilon$ being constant), $(\partial \tilde{\Phi} / \partial \varepsilon)_{J}$ is the precessional drift period, and $(\partial \varepsilon / \partial \alpha)_{J}=\langle d \beta / d t\rangle$ is the bounce-averaged rate of precession [10].

Now we turn to the calculation of the energy (44) for the flute-like perturbations, characterized by the following displacement vector $\boldsymbol{\xi}$ :

$$
\begin{gathered}
\xi=\mathbf{b} \xi_{\|}+\xi_{\perp}, \\
\xi_{\perp}=\frac{\mathbf{B} \times \nabla \eta}{B^{2}},
\end{gathered}
$$

with function $\eta$ constant along the field line,

$$
\eta=\eta(\alpha, \theta)
$$

Since

$$
\nabla \eta=\frac{\partial \eta}{\partial \alpha} \nabla \alpha+\frac{\partial \eta}{\partial \theta} \nabla \theta
$$

the perpendicular displacement (51) can be expressed as

$$
\boldsymbol{\xi}_{\perp}=-\frac{\partial \eta}{\partial \theta} \mathbf{u}+\frac{\partial \eta}{\partial \alpha} \mathbf{v}
$$

In the curved coordinate system $(\alpha, \theta, l)$ ( with $l$ as a coordinate along the field line, determined by $d l=d \chi / B$ ) one obtains:

$$
\begin{aligned}
\nabla \cdot \boldsymbol{\xi} & =B \frac{\partial}{\partial l}\left(\frac{\xi_{i}}{B}\right)+B^{2}\left[\frac{\partial}{\partial \theta}\left(\frac{1}{B^{2}} \frac{\partial \eta}{\partial \alpha}\right)-\frac{\partial}{\partial \alpha}\left(\frac{1}{B^{2}} \frac{\partial \eta}{\partial \theta}\right)\right]= \\
& =B \frac{\partial}{\partial l}\left(\frac{\xi_{\|}}{B}\right)-\frac{2}{B}\left[\frac{\partial B}{\partial \theta} \frac{\partial \eta}{\partial \alpha}-\frac{\partial B}{\partial \alpha} \frac{\partial \eta}{\partial \theta}\right] .
\end{aligned}
$$

As it follows from (46), (50), the expression for $q$ yields:

$$
q=\frac{\partial \xi_{\|}}{\partial l}-\boldsymbol{\xi}_{\perp} \cdot(\mathbf{b} \cdot \nabla) \mathbf{b}
$$

Accounting for $\nabla \times B=0$, and using the relationships

$$
\begin{gathered}
(\mathbf{b} \cdot \nabla) \mathbf{b}=-\mathbf{b} \times(\nabla \times \mathbf{b}), \\
\nabla \times \mathbf{b}=-\frac{1}{B}(\nabla B \times \mathbf{b}),
\end{gathered}
$$


we come to

$$
q=\frac{\partial \xi_{\|}}{\partial l}-\frac{1}{B}\left[\frac{\partial B}{\partial \theta} \frac{\partial \eta}{\partial \alpha}-\frac{\partial B}{\partial \alpha} \frac{\partial \eta}{\partial \theta}\right] .
$$

After simple manipulations one can find from (48), (53), (54) that

$$
\begin{gathered}
\langle H\rangle=\left(\frac{\partial J}{\partial \varepsilon}\right)^{-1} \int \frac{d l}{v_{\|}} \xi_{\|} \frac{\partial}{\partial l}\left(\frac{M v_{\|}^{2}}{2}+\mu B\right)+ \\
+\left(\frac{\partial J}{\partial \varepsilon}\right)^{-1} \int \frac{d l}{B v_{\|}}\left(M v_{\|}^{2}+\mu B\right)\left[\frac{\partial B}{\partial \theta} \frac{\partial \eta}{\partial \alpha}-\frac{\partial B}{\partial \alpha} \frac{\partial \eta}{\partial \theta}\right]
\end{gathered}
$$

The contribution from the first integral vanishes, since the particle energy conserves along the field line.

As it was shown in [15],

$$
\begin{aligned}
& \frac{\partial J}{\partial \alpha}=-\int \frac{d l}{B v_{\|}}\left(M v_{\|}^{2}+\mu B\right) \frac{\partial B}{\partial \alpha}, \\
& \frac{\partial J}{\partial \theta}=-\int \frac{d l}{B v_{\|}}\left(M v_{\|}^{2}+\mu B\right) \frac{\partial B}{\partial \theta} .
\end{aligned}
$$

Combining (56),(57) with (55), one can rewrite the expression for $\langle H\rangle$ as

$$
\langle H\rangle=-\left(\frac{\partial J}{\partial \varepsilon}\right)^{-1} \frac{\partial J}{\partial \theta} \frac{\partial \eta}{\partial \alpha}+\left(\frac{\partial J}{\partial \varepsilon}\right)^{-1} \frac{\partial J}{\partial \alpha} \frac{\partial \eta}{\partial \theta} .
$$

Now it is easy to perform the average of $\langle H\rangle$ over the drift motion. Inserting (58) into (49) and taking into account that

$$
\left(\frac{\partial \alpha}{\partial \theta}\right)_{J}=-\frac{\partial J}{\partial \theta}\left(\frac{\partial J}{\partial \alpha}\right)^{-1},\left(\frac{\partial \varepsilon}{\partial \alpha}\right)_{J}=-\frac{\partial J}{\partial \alpha}\left(\frac{\partial J}{\partial \varepsilon}\right)^{-1}
$$

we have:

$$
\langle\langle H\rangle\rangle=\left(\frac{\partial \bar{\Phi}}{\partial \varepsilon}\right)_{J}^{-1} \oint\left(d \theta \frac{\partial \eta}{\partial \theta}+d a \frac{\partial \eta}{\partial \alpha}\right)=\left(\frac{\partial \bar{\Phi}}{\partial \varepsilon}\right)_{J}^{-1} \oint d \eta=0 .
$$

Finally, using

$$
\left(\frac{\partial F}{\partial \alpha}\right)_{e}=-\left(\frac{\partial J}{\partial \varepsilon}\right)\left(\frac{\partial \varepsilon}{\partial \alpha}\right)_{J}\left(\frac{\partial F}{\partial J}\right)_{e}
$$

one obtains after the substitution of (58) into (47):

$$
W_{k}=-\frac{1}{2} \int d \alpha d \theta d \mu d \varepsilon\left(\frac{\partial J}{\partial \alpha}\right)\left(\frac{\partial F}{\partial \alpha}\right)_{e}\left(\left(\frac{\partial \eta}{\partial \theta}\right)^{2}-\right.
$$




$$
\left.-2\left(\frac{\partial J}{\partial \theta}\right)\left(\frac{\partial J}{\partial \alpha}\right)^{-1}\left(\frac{\partial \eta}{\partial \alpha}\right)\left(\frac{\partial \eta}{\partial \theta}\right)+\left(\frac{\partial J}{\partial \alpha}\right)^{-2}\left(\frac{\partial J}{\partial \theta}\right)^{2}\left(\frac{\partial \eta}{\partial \alpha}\right)^{2}\right)
$$

Here we have changed the set of the integration variables, and perform the integration over $d \varepsilon$ instead of $d J$.

Now we turn to the calculation of the energy variation $W_{f}$. Since $W_{f}$ does not depend on the parallel component $\xi_{\|}$(see [14]), and besides that the displacement (50) does not perturb the magnetic field, $Q=0$, the expression (45) reduces to

$$
W_{f}=-\frac{1}{2} \int d^{3} x\left(\frac{1}{B}\left(\xi_{\perp} \cdot \nabla B\right)\left(\xi_{\perp} \cdot \nabla^{\prime} p_{\perp}\right)+\frac{1}{B}\left(\xi_{\perp} \cdot \nabla^{\prime} p_{\|}\right)\left[\frac{\partial B}{\partial \theta} \frac{\partial \eta}{\partial \alpha}-\frac{\partial B}{\partial \alpha} \frac{\partial \eta}{\partial \theta}\right]\right) .
$$

The parallel and nerpendicular pressure components, presented in (60), are given by

$$
\begin{aligned}
& p_{\|}=\int \frac{d \varepsilon d \mu}{v_{\|}} B \quad M v_{\|}^{2} F, \\
& p_{\perp}=\int \frac{d \varepsilon d \mu}{v_{\|}} B \quad \mu B F .
\end{aligned}
$$

The substitution of (52) into (60) leads to

$$
\begin{aligned}
W_{f}=\frac{1}{2} \int \frac{d^{3} x}{B} & \left\{\frac{\partial B}{\partial \alpha}\left[\frac{\partial P}{\partial B} \frac{\partial B}{\partial \alpha}-\frac{\partial P}{\partial \alpha}\right]\left(\frac{\partial \eta}{\partial \theta}\right)^{2}+\frac{\partial B}{\partial \theta}\left[\frac{\partial P}{\partial B} \frac{\partial B}{\partial \theta}-\frac{\partial P}{\partial \theta}\right]\left(\frac{\partial \eta}{\partial \alpha}\right)^{2}-\right. \\
& \left.-\frac{\partial B}{\partial \alpha}\left[\frac{\partial P}{\partial B} \frac{\partial B}{\partial \theta}-\frac{\partial P}{\partial \theta}\right] \frac{\partial \eta}{\partial \theta} \frac{\partial \eta}{\partial \alpha}-\frac{\partial B}{\partial \theta}\left[\frac{\partial P}{\partial B} \frac{\partial B}{\partial \alpha}-\frac{\partial P}{\partial \alpha}\right] \frac{\partial \eta}{\partial \theta} \frac{\partial \eta}{\partial \alpha}\right\}
\end{aligned}
$$

where $P=p_{\|}+p_{\perp}$,

$$
P=\int \frac{d \varepsilon d \mu B}{v_{\|}}\left(M v_{\|}^{2}+\mu B\right) F(\mu, \varepsilon, J) .
$$

The calculation of the derivatives of $P$, entering into (61), gives:

$$
\begin{gathered}
\frac{\partial P}{\partial B}=\int d \varepsilon d \mu F \frac{\partial}{\partial B}\left(B \frac{\left(M v_{\|}^{2}+\mu B\right)}{v_{\|}}\right), \\
\frac{\partial P}{\partial \alpha}=\frac{\partial B}{\partial \alpha} \int d \varepsilon d \mu F \frac{\partial}{\partial B}\left(B \frac{\left(M v_{\|}^{2}+\mu B\right)}{v_{\|}}\right)+\int \frac{d \varepsilon d \mu B}{v_{\|}}\left(M v_{\|}^{2}+\mu B\right)\left(\frac{\partial F}{\partial \alpha}\right)_{e} \\
\frac{\partial P}{\partial \theta}=\frac{\partial B}{\partial \theta} \int d \varepsilon d \mu F \frac{\partial}{\partial B}\left(B \frac{\left(M v_{\|}^{2}+\mu B\right)}{v_{\|}}\right)+\int \frac{d \varepsilon d \mu B}{v_{\|}}\left(M v_{\|}^{2}+\mu B\right)\left(\frac{\partial F}{\partial \theta}\right)_{e}
\end{gathered}
$$

Now reminding that $d^{3} x=d \alpha d \theta d l / B$, and accounting for (56), (57), (62), one can transform (61) to

$$
W_{f}=\frac{1}{2} \int d \varepsilon d \mu d \alpha d \theta\left\{\frac{\partial J}{\partial \alpha}\left(\frac{\partial F}{\partial \alpha}\right)_{e}\left(\frac{\partial \eta}{\partial \theta}\right)^{2}+\right.
$$




$$
\left.+\frac{\partial J}{\partial \theta}\left(\frac{\partial F}{\partial \theta}\right)_{e}\left(\frac{\partial \eta}{\partial \alpha}\right)^{2}-\left(\frac{\partial J}{\partial \theta}\left(\frac{\partial F}{\partial \alpha}\right)_{e}+\frac{\partial J}{\partial \alpha}\left(\frac{\partial F}{\partial \theta}\right)_{e}\right) \frac{\partial \eta}{\partial \theta} \frac{\partial \eta}{\partial \alpha}\right\}
$$

The latter expression, together with equations

$$
\begin{gathered}
\left(\frac{\partial F}{\partial \theta}\right)_{e}=\frac{\partial J}{\partial \theta}\left(\frac{\partial F}{\partial J}\right)_{e},\left(\frac{\partial F}{\partial \alpha}\right)_{e}=\frac{\partial J}{\partial \alpha}\left(\frac{\partial F}{\partial J}\right)_{e} \\
\left(\frac{\partial F}{\partial \theta}\right)_{e}=\frac{\partial J}{\partial \theta}\left(\frac{\partial J}{\partial \alpha}\right)^{-1}\left(\frac{\partial F}{\partial \alpha}\right)_{e}
\end{gathered}
$$

allows one to show easily that $W_{f}$ equals to the expression (59) with the inverse sign, and hence the energy variation (44) is found to be identically zero.

\section{B Energy principle for the "disk"-like plasma}

Consider the limit of zero longitudinal hot plasma pressure, $p_{\|}=0$, when transverse energy of hot particles greatly exceeds their longitudinal energy. In the equilibrium state these particles perform small bounce oscillations round minimum field point, so that

$$
\varepsilon-\mu B_{0} \ll \mu B_{0}
$$

$B_{0}$ marks minimum value of the field strength on the given field line. Using expansion of $B$ along the field line in the vicinity of $B_{0}$,

$$
B \simeq B_{0}+\tilde{B} l^{2}
$$

one can carry out the integration in (10) explicitly:

$$
J=\pi \sqrt{2 M} \frac{\Delta \varepsilon}{\sqrt{\mu \bar{B}}}
$$

with $\Delta \varepsilon=\varepsilon-\mu B_{0}$. Now it would not be difficult to calculate with sufficient accuracy $\varepsilon$ and $\Phi$ - derivatives of $J$ :

$$
\begin{gathered}
J_{\varepsilon}=\frac{J}{\Delta \varepsilon}, \quad J_{\Phi}=-\mu B_{0 \Phi} \frac{J}{\Delta \varepsilon} . \\
J_{c \varepsilon}=0, \quad J_{e \Phi}=-\frac{\bar{B}_{\Phi}}{\bar{B}} \frac{J}{\Delta \varepsilon} . \\
J_{\Phi \Phi}=-\mu B_{0 \Phi \Phi} \frac{J}{\Delta \varepsilon}-\mu B_{0 \Phi} \frac{\bar{B}_{\Phi}}{\tilde{B}} \frac{J}{\Delta \varepsilon} .
\end{gathered}
$$

Here we have taken into account, that $\Delta \varepsilon / \mu B_{0}$ is a small parameter according to (64). Substitution of (66) into (24) leads to the following expression for the energy variation:

$$
W=\frac{e^{2}}{4} \int d \mu d \Phi \frac{F}{\mu} \frac{d}{d \Phi}\left(\frac{\bar{\ddagger}^{2}}{B_{0 \Phi}}\right) .
$$


Note that there is no integration over $d \varepsilon$ in (67). The reason is that in the case under consideration, the distribution function $F$ depends on $\varepsilon$ approximately in a $\delta$-functional way, $F \sim \delta\left(\varepsilon-\mu B_{0}\right)$, and so the integration over $d \varepsilon$ can be done explicitly.

\section{Generalization of the energy principle for the case: $\Gamma / m \ll \Omega_{d} \leq \Gamma$}

The crucial point of the derivation of the expression for the energy variation in Section 2 (as well as in [7]) is the exploitation of the fact that the flux adiabatic invariant conserves. As is well known (see, e.g. [10]), when the frequency of the drift motion $\Omega_{d}$ around the axis is high as compared with the inverse characteristic time of variation of the electric and magnetic fields, then the magnetic flux $\bar{\Phi}$ encompassed by the drift surface is an adiabatic invariant (notation $\tilde{\Phi}$ shouldn't be confused with a flux coordinate $\Phi$, an independent variable). In the case considered in the present paper, the magnetic field is constant; the varying is electrostatic potential $\varphi$ of the perturbations. The slowness of variation of $\varphi$ in the above mentioned sense (see (4)) guarantees the conservation of $\bar{\Phi}$.

When considering the case of perturbations with high azimuthal mode number $m \gg 1$, one may encounter the situation when the drift frequency $\Omega_{d}$ is lower then $\Gamma$ but higher than $\Gamma / m$ :

$$
\Gamma / m \ll \Omega_{d} \leq \Gamma .
$$

The inequality $\Omega_{d} \leq \Gamma$ means that one cannot thoughtlessly use the traditional adiabatic invariant $\bar{\Phi}$. However, we shall show that, under the condition $\Gamma / m \ll \Omega_{d}$, there exists another adiabatic invariant, similar to $\bar{\Phi}$.

We consider the potential perturbation (not necessarily small) which is of the form (8) and changes in time with the characteristic frequency $\Gamma$. Then the inequality $\Gamma / m \ll \Omega_{d}$ means that the guiding centre traverses one spatial period of the system in a time that is short as compared to the time of potential variation. Just this fact is a basis for existence of the generalized adiabatic invariant.

The guiding centre motion, averaged over the fast bouncing along the field line, is governed by the following equations [11]:

$$
\begin{gathered}
\dot{\psi}=-\frac{2 \pi c}{e t_{\|}} J_{\Phi}, \\
\dot{\Phi}=\frac{2 \pi c}{e t_{\|}} J_{\psi},
\end{gathered}
$$

where $t_{\|}=J_{e}$ is the transit time between the turning points. In the context of the problem under consideration, $J$ is a constant of motion (as well as $\mu$ ). Resolving the equation $J=$ const with respect to $\varepsilon$, we can find the function $\varepsilon(\psi, \Phi)$ for a given particle. By differentiating the function $J(\varepsilon(\psi, \Phi), \Phi, \psi)$ over $\psi$ and $\Phi$, we find:

$$
\frac{\partial \varepsilon}{\partial \psi}=-\frac{J_{\psi}}{J_{\varepsilon}}, \quad \frac{\partial \varepsilon}{\partial \Phi}=-\frac{J_{\Phi}}{J_{\varepsilon}}
$$


and the equations of motion (69) acquire the Hamiltonian form:

$$
\begin{gathered}
\dot{\psi}=\frac{2 \pi c}{e} \frac{\partial \varepsilon}{\partial \psi} \\
\dot{\Phi}=-\frac{2 \pi c}{e} \frac{\partial \varepsilon}{\partial \psi}
\end{gathered}
$$

with $\psi$ and $\Phi$ being canonically conjugate variables and $\varepsilon$ playing a role of Hamiltonian. As the explicit dependence of $\varepsilon$ on $t$ is slow in the sense that the guiding centre traverses one spatial period of the system (in coordinate $\psi$ ) in a time short as compared to the time of potential variation, equations $(70)$ possess an adiabatic invariant

$$
\bar{\Phi}^{\star}=\int_{T_{\psi}} \Phi d \psi
$$

where integration is carried out over one spatial period $T_{\psi}$, equal to $2 \pi / \mathrm{m}$. Of course, for slow enough potential variations, $\Gamma \ll \Omega_{d}$, the integration in (71) can be extended to a full rotation of a particle around the axis, and (71) reduces to the standard flux invariant.

Now it becomes clear that the expression for the energy variation, that takes into account conditions (68), coincides with formula (24). Indeed, all calculations in this case would exactly repeat calculations, performed in Section 2 , with the only exclusion: $\tilde{\Phi}$ conservation requirement should be replaced by the requirement of the conservation of $\bar{\Phi}^{\star}$. But this changing doesn't, obviously, affect formula (24), since the averaging of the expressions, composed of function (8), either over $2 \pi / m$-interval (as in (71)) or over $2 \pi$-interval (as in the expression for $\tilde{\Phi})$ would finally lead to the same result.

\section{References}

[1] D.D. Ryutov, G.V. Stupakov, JETP Lett., 42, 29 (1985).

[2] D.D. Ryutov, G.V. Stupakov, Sov.J. Plasma Phy.s.. 12. 815 (1986).

[3] G.I. Dimov, Proceedings of 10th European Schoul on Plasma Phỵsics, Tbilisi, 157 (1991).

[4] M.N. Rosenbluth, N.A.Krall, N.Rostoker, Nucl. Fusion. Suppl. Pt. 1, 143 (1962).

[5] M.D. Kruskal, C.R. Oberman, Phys. Fluids, 1. 275 (1958).

[6] V.P. Nagornyj, D.D. Ryutov, G.V. Stupakov, Preprint X-83-74. Institute of Nuclear Physics, Novosibirsk (1983).

[7] J.W. Van Dam, M.N. Rosenbluth, Y.C. Lee. Phỵ.s. Fluids. 25.1349 (1982).

[8] I.M. Lansky, D.D. Ryutov, JETP Letters, 57. No.1. 30 (1992). 
[9] F.H. Coensgen, T.A. Casper, D.L. Correl, et al., Rep. UCRL-97280, Rev. 1, Lawrence Livermore National Laboratory (1987).

[10] T.G. Northrop, . . Teller, Phyo. Rev., 117, 215 (1960).

[11] A.I. Morozov, L.S. Solov'ev, Reviews of Plasma Physics, 2, Consultants Bureau, New York (1966).

[12] D.D. Ryutov, G.V. Stupakov, Reviews of Plasma Physics, 13, Consultants Bureau, New York - London, 93 (1987).

[13] V.P. Nagornyj, D.D. Ryutov, G.V. Stupakov, Nucl. Fusion, 24, No. 11, 1421 (1984).

[14] J.B. Taylor, R.J. Hastie, Phys.Fluids, 8, 232 (1965).

[15] W.A. Newcomb, J. Plasma Physics, 26, part 3, 529 (1981).

[16] R.J. Hastie, J.B. Taylor, F.A. Haas, Ann.Phys. (NY), 41, 302 (1967).

[17] V.V. Mirnov, V.P. Nagornyj, D.D. Ryutov, Preprint N-84-40, Institute of Nuclear Physics, Novosibirsk (1984). 

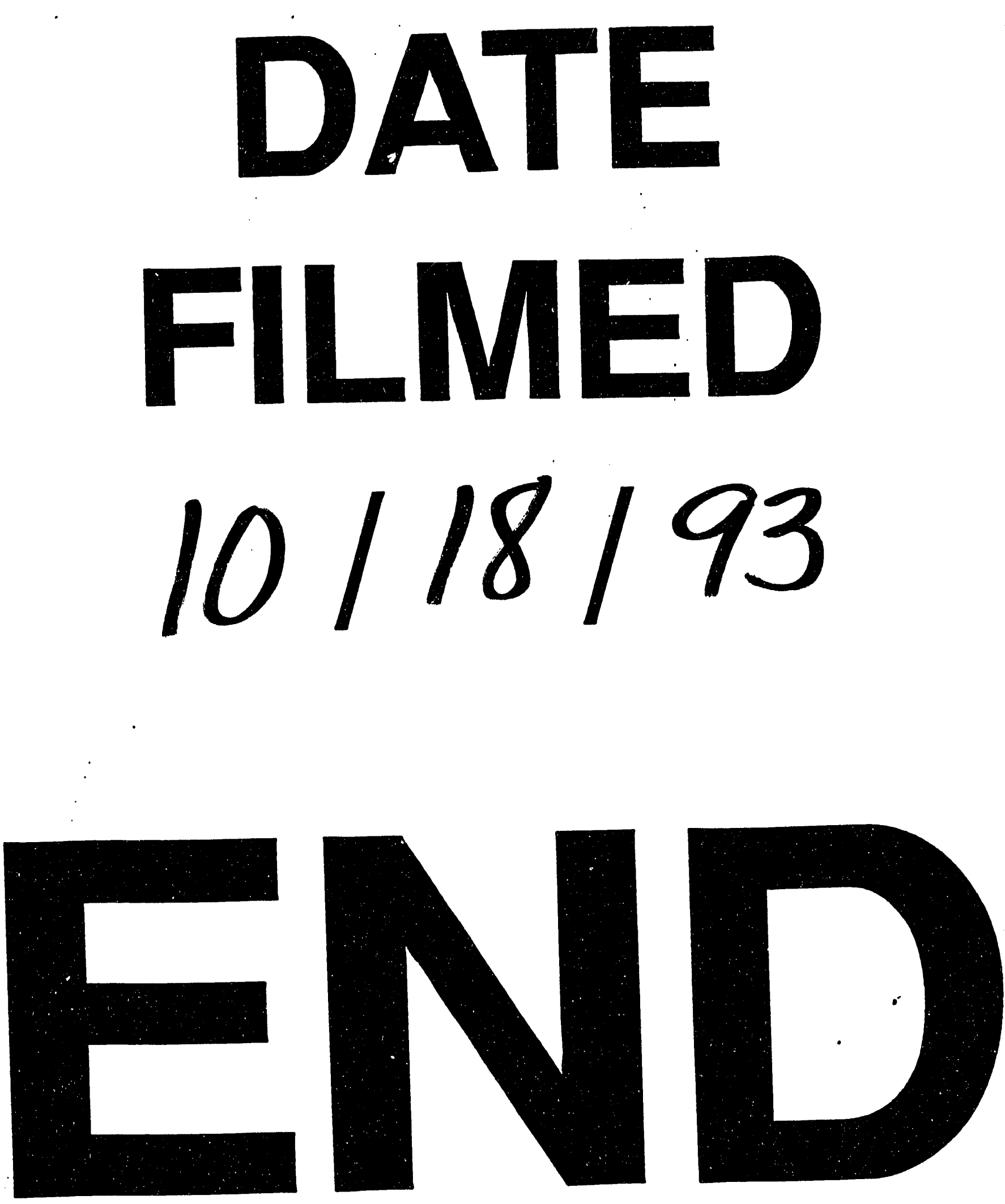
